\title{
Continuous monitoring of cortical perfusion by laser Doppler flowmetry in ventilated patients with head injury
}

\author{
Peter J Kirkpatrick, Pieotr Smielewski, Marek Czosnyka, John D Pickard
}

\begin{abstract}
A method for monitoring cortical perfusion by laser Doppler flowmetry (LDF) in the neurointensive care unit is described. Out of 22 patients with head injuries, reliable and long term recordings were obtained in 16. Laser Doppler flowmetry registered changes in cortical microcirculatory flow in response to spontaneous waves of raised intracranial pressure, and to therapeutic manoeuvres that altered the cerebral perfusion pressure. Comparisons of variations in flux signal with cerebral perfusion pressure provided an indication of the autoregulatory state of the cortical microcirculation, and analysis of raw LDF data demonstrated an autoregulatory breakpoint of cerebral perfusion pressure of $58 \mathrm{~mm} \mathrm{Hg}$, below which cortical perfusion failed. Although middle cerebral artery flow velocities were generally tightly coupled with LDF signal changes, episodes of uncoupling were seen. The potential uses and limitations of LDF in the neurointensive care setting are discussed.
\end{abstract}

(F Neurol Neurosurg Psychiatry 1994;57:1382-1388)

The secondary pathophysiological mechanisms excited after brain trauma are complex, and involve dynamic changes in intracranial pressure, ${ }^{1}$ cerebral perfusion pressure, ${ }^{2}$ and cerebral blood flow. ${ }^{3}$ In conditions of low cerebral perfusion pressure, varying degrees of impaired autoregulation of cerebral blood flow occur that may compromise oxygen and substrate delivery to the brain parenchyma. ${ }^{245}$ Such secondary events are believed to be important in the generation of ischaemic lesions seen after severe head injury. ${ }^{6}$

The recognition of a changing state of cerebral perfusion pressure in patients with head injuries has required the continuous monitoring, recording, and computer processing of data derived from reliable intracranial and arterial pressure measuring devices. Noninvasive transcranial Doppler studies allow waveform analysis of blood flow velocities in the basal arteries. Falling cerebral perfusion pressure is associated with changes in the flow velocity pulsatility indices that are believed to reflect failing cerebral autoregulation. ${ }^{37}$ The effect of a changing cerebral perfusion pressure, however, on the parenchymal microcirculatory flow in patients with head injuries is largely unknown. Classic methods used to estimate regional cerebral blood flow clinically, such as the ${ }^{133}$ xenon clearance method, and xenon enhanced CT, have limited temporal resolution, ${ }^{38}$ hence changes in regional cerebral blood flow accompanying transient alterations in cerebral perfusion pressure have proved difficult to capture. A method providing a continuous measure of cerebral blood flow that can be established within the neurointensive care unit is needed.

Laser Doppler flowmetry (LDF) is a technique that provides a continuous measure of relative microcirculatory flow..$^{10}$ The final signal generated is a measure of microcirculatory red cell flux (the product of red cell concentration and the red cell velocity). ${ }^{112}$ Experimental use of LDF in vitro ${ }^{13-15}$ and in vivo ${ }^{13}{ }^{15-20}$ has consistently demonstrated a close linear correlation between LDF flux and cerebral perfusion pressure measured with a variety of standard methods. The method has shown particular use in the observation of changes in microcirculatory flow induced by physiological and pharmacological stimuli, ${ }^{1821-27}$ and the laser light used does not seem to alter the morphological and physiological characteristics of the vascular bed examined. ${ }^{18}$

Laser Doppler flowmetry is not quantitative, however, records from a small tissue volume, and provides no information on the direction of blood flow. Furthermore, experience has shown that the flux signal is very sensitive to the artifacts of local tissue pressure and movement, so that the reliability of the technique is critically dependent on the method of application. Despite these drawbacks, LDF has already shown potential for blood flow measurements in several clinical disciplines including dermatology, ${ }^{28}$ vascular surgery, ${ }^{29}$ and ophthalmology. ${ }^{30}$ In neurosurgery, some success has been reported in the intraoperative assessment of cortical blood flow during general neurosurgical procedures, ${ }^{31}$ during surgery for superficial arteriovenous malformations, ${ }^{32}$ and for defining the characteristics of blood flow through various cerebral tumours. ${ }^{33}$ Long term bedside monitoring of regional cerebral blood flow in comatose patients with flexible implanted optical probes has also recently been achieved by Meyerson et al. ${ }^{34}$

Using a rigid LDF probe, we present a relatively simple and safe procedure for monitoring cortical flux in the neurointensive care unit. The data derived from long term monitoring in 22 patients with severe head injuries has been analysed. The findings indicate that 
Figure 1 Laser Doppler P3 probe and adapted Camino support bolt. in $5 \mathrm{ml}$ of motility standard (Moor Instruments Ltd). Sterilisation was achieved by immersion in $2 \%$ glutaldehyde solution for 30 minutes (Cidex, ASEP, UK) with subsequent washing in sterile saline.

\section{PATIENTS}

Over a nine month period, 22 patients (aged 19-69) with moderate to severe head injuries (Glasgow coma score 3-12) were entered into the study. The study was approved by the Cambridge Health Authority local research ethics committee.

\section{PATIENT MANAGEMENT}

All patients were sedated with midazolam

LDF registers changes in cortical microcirculatory flow during changing intracranial pressure and cerebral perfusion pressure. The response to ventilator adjustments and manipulation of the arterial blood pressure with dopamine can also be monitored with LDF. Further, group analysis of the LDF signal data has supported the concept of an autoregulatory threshold of cerebral perfusion pressure below which cerebral perfusion fails. Problems in providing reliable flux signals, avoidance of artifacts, and difficulties with quantification and comparison of signal changes during long term monitoring are discussed.

\section{Patients and methods}

The theoretical basis for LDF is complex. ${ }^{11} 12$ Briefly, monochromatic coherent laser light, delivered via a transmitting fibre optic, is scattered by biological tissues. Light scattered from moving structures (red blood cells) experiences a Doppler shift in frequency (shifted light), whereas that reflected from surrounding stationary structures remains unaltered (reference light). The shifted and reference light signals are collected by a receiving afferent fibre optic and detected on the surface of a photodetector. The interference of the two signals produces optical beating (heterodyning), the frequency of which is equal to the Doppler shifted frequency. A spectrum of shifted frequencies is generated from variations in red cell velocities, and variations in the angle of incidence of light on red cell surfaces. The power spectral density of these shifted frequencies is determined by the red cell concentration and velocity. From the alternating photodetector current, various algorithms relating red cell flux to output signal can be derived. ${ }^{12}$

The MBF3D dual channel laser Doppler flowmeter (Moor Instrument Ltd, Devon UK) adopted in this study uses a low energy $(0.5-1.5 \mathrm{~mW})$ laser light generated by a semiconductor laser diode in the near infrared spectrum (wavelength $=780-820 \mathrm{~nm}$ ). The signal processor provides variable recording bandwidths of $3 \cdot 1,14 \cdot 9$, and $22 \cdot 4 \mathrm{kHz}$. In the present study a recording frequency of $14 \cdot 6$ $\mathrm{kHz}$ was adopted with a Moor P3 probe (fig 1).

Before use, the probes were precalibrated
$(2-10 \mathrm{mg} / \mathrm{h}$ infusion) and fentanyl $(0 \cdot 1-0.5$ $\mathrm{mg} / \mathrm{h}$ infusion), paralysed with atracurium $(0.3-1.2 \mathrm{mg} / \mathrm{kg} / \mathrm{h}$ infusion), intubated, and ventilated to a $\mathrm{PCO}_{2}$ of $3.5-4.0 \mathrm{kPa}$. Intravenous fluid (Haemaccel and normal saline) were given to achieve a central venous pressure of between $5-10 \mathrm{~cm} \mathrm{H}_{2} \mathrm{O}$. Attempts were made to maintain a cerebral perfusion pressure of greater than $55 \mathrm{~mm} \mathrm{Hg}$ in those patients with raised intracranial pressure using a constant infusion of dopamine (5-15 $\mu \mathrm{g} / \mathrm{kg} / \mathrm{min}$ ). If this treatment failed, boluses of mannitol ( $200 \mathrm{ml}$ of $20 \%$ over 20 minutes) were given and repeated as necessary.

For assistance with the interpretation of data, the nursing staff were asked to document and time any manoeuvre that might cause a signal artifact (such as turning the patient, endotracheal suction, flushing of arterial lines). They were also invited to perform their tasks during specific periods, providing two-hour intervals for the collection of relatively undisturbed data.

\section{MONITORING}

Invasive and continuous monitoring of arterial blood pressure (20 G catheters, Arrow UK; transducers and monitors, $S$ and $W$ Denmark) and intracranial pressure (Camino, USA) were routinely undertaken in all patients. The intracranial pressure supporting bolt was positioned in the right or left frontal region according to the side of maximum injury as seen on admission CT. In 10 patients, long term monitoring of middle cerebral artery flow velocities was also undertaken by transcranial Doppler (TCD-Scimed, UK).

\section{LASER DOPPLER FLOWMETRY}

At the time of insertion of the Camino intracranial pressure device, a second Camino bolt was sited about $3 \mathrm{~cm}$ further lateral to the intracranial pressure bolt to support a single LDF probe. Care was taken during the siting of this bolt not to breach the dura with the twist drill such that once the drill hole was completed, a blunt stylet passed down the hole could be felt to spring upon the dura. The camino bolt was then twisted in position, and the dura was punctured with a lumbar needle passed down the bolt shaft. If any bleeding occurred, haemostasis was secured by irrigation with normal saline until the 
effluent was clear. A precalibrated and sterilised LDF probe was passed through the support bolt until the pliable surface of the cortex was encountered. With the probe connected to the monitor, the probe position was withdrawn (2-3 mm) until a maximal pulsatile signal was achieved. On further withdrawal (1-2 $\mathrm{mm}$ ) the signal began to fall. The depth of the probe was increased again until the maximum signal was regained, at which point the locking screw was tightened. Repositioning of the probe was possible once the patient was on the neurointensive care unit allowing intermittent optimisation of the signal.

In four cases, intraoperative placement of the LDF probe indicated that the cortex had been breached (thereby offering little resistance to the LDF probe as it penetrated into the brain parenchyma). Two of these occurred as a result of the twist drill inadvertently penetrating the dura. As the probe tip entered the brain parenchyma, the intensity of the signal fell to about $30 \%$ of the more superficial flux readings. To achieve a reliable surface cortical flux signal, the probes had to be resited.

SIGNAL CAPTURE AND PROCESSING

Signals of arterial blood pressure, intracranial pressure, LDF, and LDF amplitude were sampled at a frequency of $40 \mathrm{~Hz}$, and digitised with a 12 bit analog to digital converter (DT 2814, Data Translation, USA). Arterial blood pressure and intracranial pressure were calibrated in appropriate units $(\mathrm{mm} \mathrm{Hg}$ ), whereas the raw LDF signal was recorded in arbitrary units (0-1000 AU). Waveforms were processed with specific software (ICM; M Czosnyka, University of Cambridge). A minute by minute graphical display of mean cerebral perfusion pressure, intracranial pressure, LDF, and middle cerebral artery flow velocity (in 10 patients) was provided to assist in the clinical management of the individual patients. High frequency noise and artifacts were filtered, and signals averaged over consecutive 3 second periods. Pulse waveforms of arterial blood pressure, intracranial pressure, and LDF were evaluated with a spectral analysis algorithm to determine the amplitudes of the fundamental signals and heart rate detected. Collection of data continued until the patient was withdrawn from the ventilator $(n=12)$, had reached a static cerebral haemodynamic state $(n=8)$, or had died $(n=2)$.

\section{DATA ANALYSIS}

Time averaged raw LDF data from 16 patients in whom a reliable and continuous LDF signal had been recorded were imported into a statistical package (Statgraphics, 6+, Manugistics, USA) and the relations between LDF signal and mean cranial perfusion pressure evaluated by analysis of variance (ANOVA) within a cerebral perfusion pressure range of $30-80 \mathrm{~mm} \mathrm{Hg}$. Breakpoints for decreases in the LDF signals were defined at cerebral perfusion pressure levels below which the LDF signals started to decrease significantly $(\mathrm{p}<0.05)$.

\section{Results}

COMPLICATIONS

No complications resulted from either intracranial pressure or LDF probe insertion apart from the need to reinsert the probe in two cases. No case of CSF infection occurred. Hence although our initial cautious policy was to insert the intracranial pressure and $\mathrm{LDF}$ probes in theatre, we have identified no reason why they should not be inserted within the neurointensive care setting.

\section{RELIABILITY}

In 14 patients, a continuous pulsatile LDF signal was obtained throughout the period of monitoring. Repositioning of the probe by 1-2 mm did not alter the signal significantly
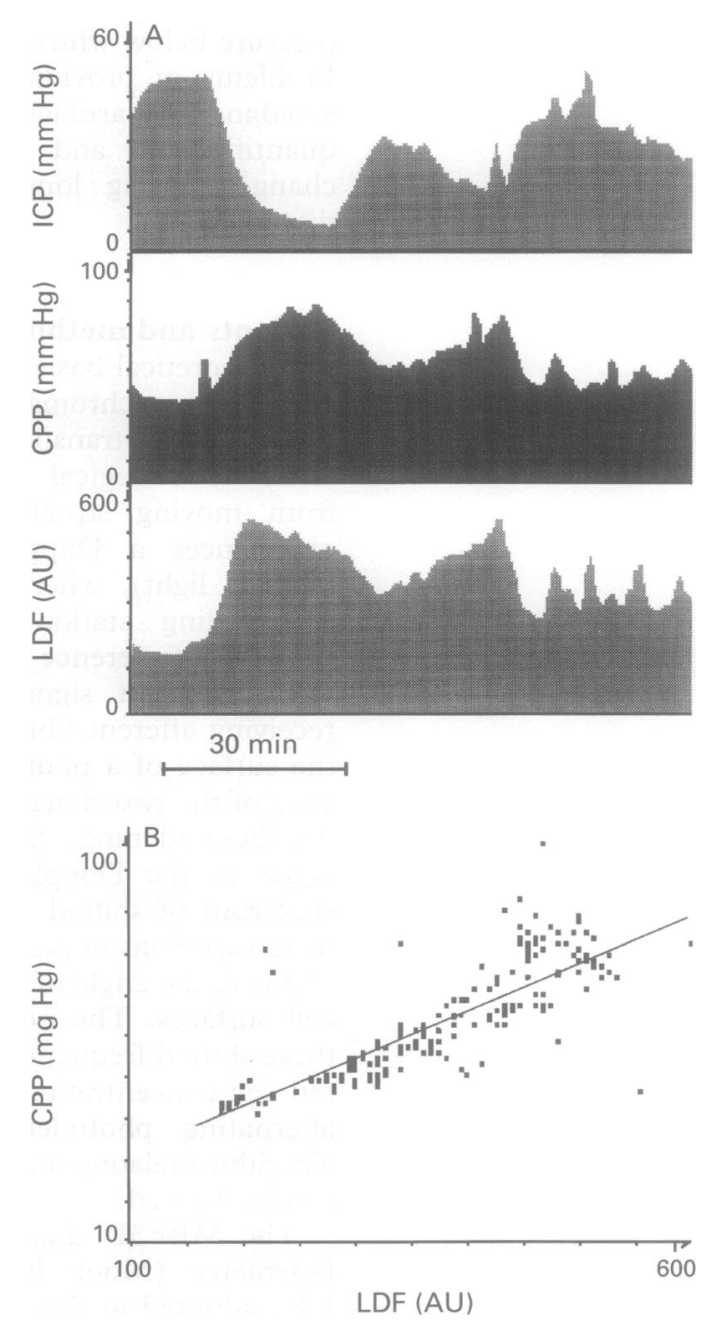

Figure 2 (A) Continuous recordings of ICP, CPP, and $L D F$ signals during waves of raised ICP in a patient with a diffuse head injury. Variations in cortical perfusion measured with $L D F$ were closely coupled to changes in $C P P$. (B) Regression analysis of the LDF and CPP signal data from the period of recording shown in $(A)$. A close correlation between $C P P$ and $L D F$ indicated that the cortical microcirculatory flow was in a state of noncortical microcirculatory flow was in a state of non-
autoregulation. ICP = intracranial pressure $(\mathrm{mm} \mathrm{Hg})$; $C P P=$ cerebral perfusion pressure $(\mathrm{mm} \mathrm{Hg}) ; L D F=$ laser Doppler flow signal (arbitrary units; $A U$ ). 
Figure 3 Upper and lower limits of $S D$ for the mean LDF signal $v$ CPP derived from analysis of raw data from long term recordings in 16 patients. The mean LDF signal shows a breakpoint at a CPP of $58 \mathrm{~mm} \mathrm{Hg}$ below which cortical perfusion falls significantly $(p<0.05)$.

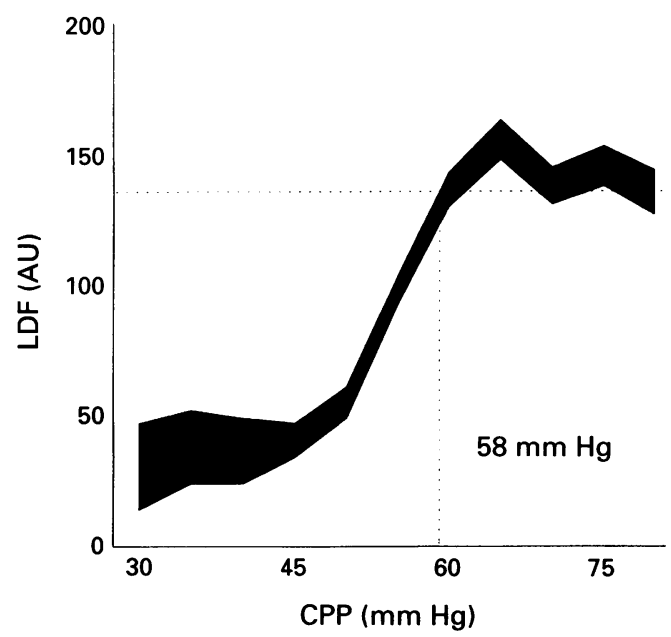

in these patients. In six other patients, the LDF signal was unreliable. Frequent adjustments to the probe position was needed to obtain a pulsatile signal, which would often disappear after a few hours. Finally, in two patients, the LDF signal could not be found on return to the intensive care unit despite several attempts to reposition the probe. On resiting the probes in theatre, continuous LDF signals were obtained in both cases.

\section{EFFECT OF LOW CEREBRAL PERFUSION}

PRESSURE ON LDF SIGNALS

At cerebral perfusion pressure values of below $30 \mathrm{~mm} \mathrm{Hg}$, the LDF signal often fell below $50 \mathrm{AU}$ and became non-pulsatile. Such low levels of cerebral perfusion pressure were recorded in five patients, two of whom died. Under such low states of cortical perfusion wide variations in non-pulsatile signal intensities occurred that were thought to be artifactual and were excluded from final data analysis (see discussion).

\section{RELATION BETWEEN CORTICAL FLUX AND} CEREBRAL PERFUSION PRESSURE

Figure 2A shows an example of a recording captured from one patient with a severe diffuse head injury during spontaneous changes in cerebral perfusion pressure. Fluctuations in intracranial pressure resulted in variations in cerebral perfusion pressure that were accompanied by changes in the LDF signal. Regression analysis showed a linear relation between cerebral perfusion pressure and LDF $(r=0.83)$ indicating a state of impaired cortical autoregulation.

Long term recordings showed variation in the autoregulatory state between patients and with time in the same patient. Analysis of the relation, however, between the raw LDF signal and cerebral perfusion pressure from all 16 patients in whom long term monitoring was achieved indicated an autoregulatory breakpoint (fig 3). The upper and lower limits of the SEM for LDF signals at varying levels of cerebral perfusion pressure $(30-80 \mathrm{~mm}$ $\mathrm{Hg}$ ) indicated a breakpoint of cerebral perfusion pressure $(58 \mathrm{~mm} \mathrm{Hg})$ at which LDF

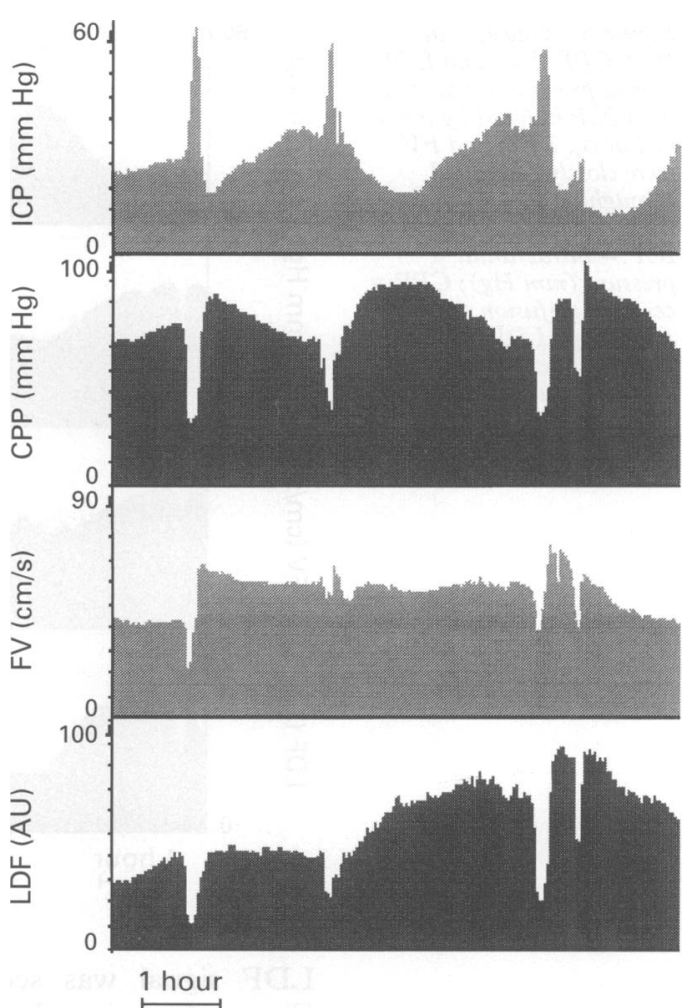

Figure 4 Recording of ICP, CPP, FV, and LDF from a patient with severe head injury. Waves of raised ICP caused a drop in CPP which resulted in impaired cerebral perfusion (as indicated by the fall in FV and $L D F$ signals). Changes in FV and LDF were closely related ( $r$ $=0 \cdot 84) . F V=$ middle cerebral artery flow velocity $(\mathrm{cm} / \mathrm{s})$. $I C P=$ intracranial pressure $(\mathrm{mm} \mathrm{Hg}) ; C P P=$ cerebral perfusion pressure ( $\mathrm{mm} \mathrm{Hg}$ ); $L D F=$ laser Doppler flow signal (arbitrary units; $A U$ ).

fell significantly on further reduction of the cerebral perfusion pressure $(p<0 \cdot 05)$. No change in LDF amplitude was detected within this range of cerebral perfusion pressure.

\section{RELATION BETWEEN CORTICAL FLUX AND} MIDDLE CEREBRAL ARTERY FLOW VELOCITY Figure 4 shows a recording captured during spontaneous wave activity of raised intracranial pressure. Periodic falls in cerebral perfusion pressure and middle cerebral artery flow velocity are seen indicating impaired perfusion in the middle cerebral artery territory. This was accompanied by a simultaneous fall in red cell flux signal. Regression analysis showed a close correlation between middle cerebral artery flow velocity and flux signals $(r=0 \cdot 84)$, confirming tight coupling of large vessel flow velocity and small vessel red cell flux.

During planned withdrawal from the ventilator in one patient, the $\mathrm{PCO}_{2}$ was allowed to rise from $4.0 \mathrm{kPa}$ to $4.8 \mathrm{kPa}$. After this manoeuvre, plateau waves of raised intracranial pressure resulted in a reduced cerebral perfusion pressure (fig 5). A tightly coupled fall in flow velocity and LDF signal $(r=0.80)$ indicated impaired cerebral and cortical perfusion. Withdrawal from the ventilator was thus delayed for a further 48 hours when a similar challenge caused no fall in perfusion at a $\mathrm{PCO}_{2}$ compatible with self ventilation.

Uncoupling between flow velocity and the 
Figure 5 Changes in $I C P, C P P, F V$, and $L D F$ during prolonged waves of high ICP induced by a rise in $\mathrm{PaCO}_{2}$. LDF and $\mathrm{FV}$ were closely correlated throughout the duration of the recording ( $r=0.80)$. ICP = intracranial pressure $(\mathrm{mm} \mathrm{Hg}) ; C P P=$ cerebral perfusion pressure $(\mathrm{mm} \mathrm{Hg}) ; \mathrm{LDF}=$ laser Doppler flow signal (arbitrary units; $A U$ ).
Figure 6 An abrupt rise in ICP and fall in CPP recorded during attempted withdrawal from inotropic (dopamine) support. Simultaneous falls in FV and LDF occurred. On recovery of CPP after recommencing inotropic support, a transient hyperaemia was registered by $L D F$.

ICP=intracranial pressure $(\mathrm{mm} \mathrm{Hg}) ; C P P=$ cerebral perfusion pressure ( $\mathrm{mm}$ $\mathrm{Hg}) ; L D F=$ laser Doppler flow signal (arbitrary units; $A U)$.
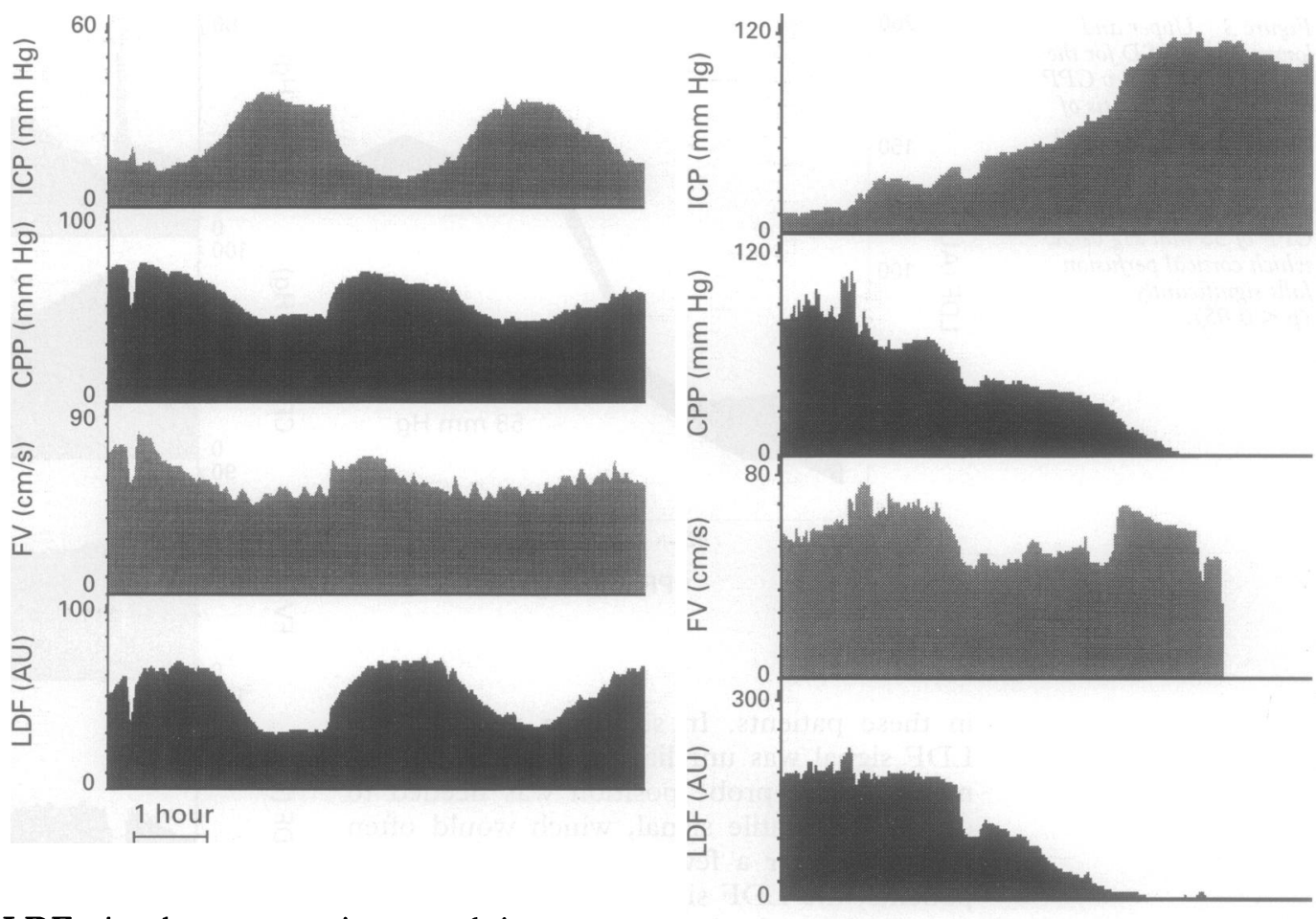

LDF signal was seen in several instances. Figure 6 shows that attempted withdrawal from dopamine caused a sudden increase in intracranial pressure and a fall in cerebral perfusion pressure. Both middle cerebral artery flow velocity and LDF signals simultaneously fell indicating impaired perfusion. On restarting the dopamine and recovering the cerebral perfusion pressure, flow velocity returned to previous levels, whereas LDF indicated a pronounced cortical hyperaemia lasting $25 \mathrm{~min}$ utes. Figure 7 shows the events of a terminal cone in another patient. During the final

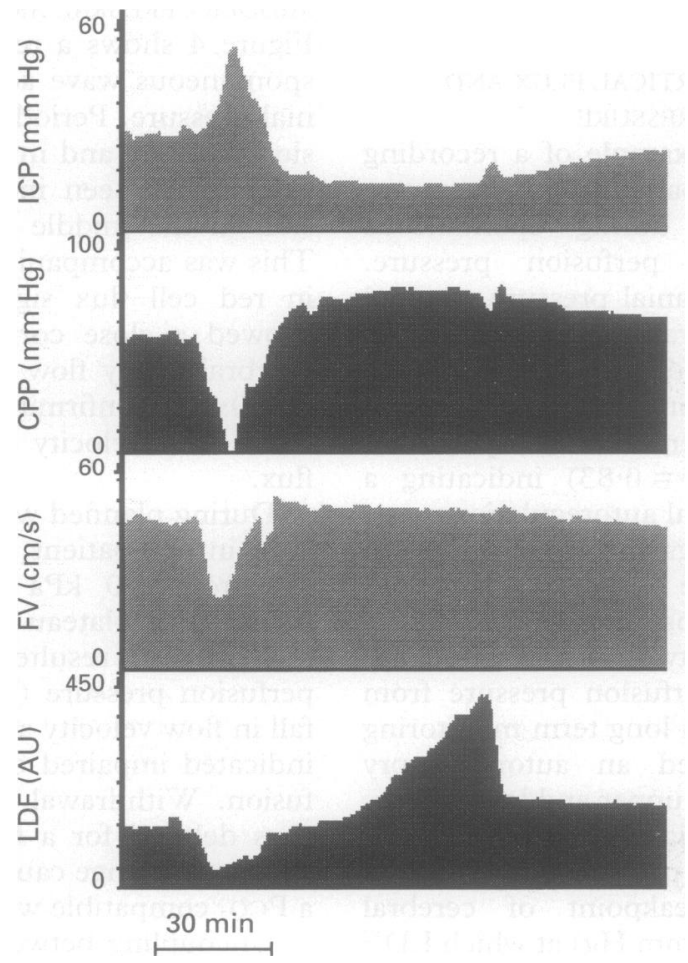

Figure 7. Progressive fall in CPP during cerebral coning in a patient with head injury who did not respond to treatment. The progressive failure of microcirculatory flow is seen in the decline in the LDF signal. FV remained positive, and later became reverberant.

hours before brain death, monitoring indicated a gradual increase in intracranial pressure despite maximum treatment. As cerebral perfusion pressure fell toward zero, the LDF signal also fell towards zero indicating failing cortical perfusion. Flow velocity recordings still registered a positive middle cerebral artery blood flow pattern which later became reverberant.

\section{Discussion}

The advantage of LDF over other methods adopted for measurement of blood flow is the high temporal resolution provided. In cerebrovascular pathology, this facility is particularly important, as a fall in cerebral blood flow lasting only a few minutes can cause irreversible ischaemic lesions. The potential use of $\mathrm{LDF}$ in monitoring cortical blood flow has been shown in individual long term recordings from 16 patients with head injuries. Thus by comparing variations in cerebral perfusion pressure with the flux signal, a real time assessment of the autoregulatory state of the cortical microcirculation was possible. Further, such recordings may prove of value when considering general therapeutic manoeuvres to improve cortical flow. For example, in one patient, the withdrawal of isotropic support caused a rapid fall in cortical perfusion, and in another, a similar event occurred after attempted withdrawal from the ventilator.

In most instances, changes in cortical perfusion measured with LDF were tightly 
coupled to changing middle cerebral artery flow velocity. These findings lend support to the use of transcranial Doppler in patients with head injuries, indicating that variations in flow velocity usually reflect variations of blood flow through the cortical microcirculation. Uncoupling of flow velocity and LDF signals were also noted, however. The hyperaemic response that followed manipulation of a dopamine infusion was registered only by LDF, indicating a possible differential effect of the drug on different parts of the vascular tree. A wider experience using combined transcranial Doppler and LDF may help to determine the incidence and relevance of such events where uncoupling occurs between large vessel and small vessel perfusion.

The technique of LDF is subject to several artifacts that are difficult to overcome in the clinical setting. Maintenance of constant probe position is particularly difficult. The sampling volume is small $\left(1-2 \mathrm{~mm}^{3}\right),{ }^{13,10}$ and may not give clinically relevant information in cases of focal pathology. Finally, LDF does not provide quantitative data. These points are considered further.

\section{LDF ARTIFACT}

By rigid fixation of an LDF probe in a position that samples from the surface of the brain, we have been able to record cortical red cell flux over long periods of time in ventilated patients with head injuries. Such monitoring is only possible in paralysed patients as movement significantly affects laser scatter, and hence output signal. Any movement incurred by nursing manoeuvres causes substantial variations in flux signal. The documentation of such events, and their restriction to allocated periods when possible, was an essential part of the study.

Unfortunately, unlike recordings in experimental animals, ${ }^{35}$ LDF through the intact dura did not provide an adequate LDF signal in our patients. The probe was therefore positioned adjacent to the pia within the subdural space. The major concern regarding data collected with this arrangement is the effect of pressure on the cortex from an inflexible probe. Variations in cerebral swelling might impinge on the probe end, thereby reducing capillary perfusion in that area and give an artificially low reading. No significant change in the LDF amplitude was noted, however, in any recording during raised intracranial pressure. Further, adjusting the depth of probe penetration into the cranial cavity by $2-3 \mathrm{~mm}$ did not significantly affect the signal intensity. Additional penetration $(5-6 \mathrm{~mm})$ caused a fall in LDF output. We interpret this as indicating that the initial probe position in these patients was $2-4 \mathrm{~mm}$ above the cortical surface without significant impingement, and that this position was maintained throughout the period of the recording.

At low levels of cerebral perfusion pressure ( $<30 \mathrm{~mm} \mathrm{Hg}$ ), LDF signals often became non-pulsatile and showed wide variations in signal intensity. These findings are likely to represent a signal artifact resulting from mechanically induced oscillations in red cell movement and reverberant flow patterns. Because LDF cannot discriminate flow direction, excessively large signal changes may result by summation of artifact.

\section{RELIABILITY OF THE METHOD}

In six patients, the LDF signal proved unreliable despite frequent attempts to adjust the depth of probe penetration. A number of explanations can account for this. Firstly, the probe may have been overlying injured brain. Secondly, inadvertent breach of the cortical surface may cause local injury that will impair red cell perfusion and cause local haemorrhage. Thirdly, the laser scatter theory of LDF assumes a small parenchymal concentration of red cells. ${ }^{15}$ Should the probe lie above a medium or large sized vessel, these assumptions are not satisfied. Finally, significant cerebral swelling may impair perfusion by the mechanisms outlined. The fact that we were able to resite the probes successfully in two patients indicates that attention to probe position with respect to the cortical surface is critical if $\mathrm{LDF}$ is to provide reliable data. It is probable that these difficulties may be partly overcome with improved probe design and technique of insertion.

\section{PROBE LOCATION}

Sampling purely from the grey matter would seem an advantage, providing information on sub-pial collateral cortical flow. The flux values would also be devoid of multicompartmental influences. Inadvertent penetration of the pia during insertion often resulted in a drop of flux signal by up to $60 \%$. This could reflect cortical injury, but may also indicate white matter flow. Long term recordings with the probe in this position were not undertaken in this study so we cannot decipher these findings further. Except for the characteristic "feel" of the probe on the surface of the brain, the methods did not allow us to be precise about the location of the probe tip. Standardisation of probe design, insertion, and calibration will be of the utmost importance in the further development of the method.

\section{SAMPLE VOLUME OF LDF}

A sample volume of $1-2 \mathrm{~mm}^{3}$ indicates that LDF registers flow changes only within the cortical grey matter. Despite a restricted sample volume, the long term recordings showed the expected pathophysiological responses to raised intracranial pressure. Further, in cases where transcranial Doppler recordings were also captured, a close correlation between middle cerebral artery flow velocity and cortical perfusion was seen indicating that changes in flow measured with LDF were representative of cortical perfusion in the general territory of the ipsilateral middle cerebral artery. Although LDF will not resolve focal anomalies in cerebral blood flow, the technique may monitor general changes that are an important component of secondary mechanisms in brain injury. 
QUANTIFICATION OF LDF

Quantification of LDF has been hampered by the variation in baseline signals produced between readings. Our own findings with LDF in experimental animals have shown that, despite a very close correlation between cortical flux and cerebral blood flow, the stable state LDF baseline readings can vary considerably between animals. This partly results from variations in the "biological zero" output signal that the instrument records when blood flow is zero. ${ }^{35}$ In our patients with head injuries, we noticed that LDF signal levels for cerebral perfusion pressure above $65 \mathrm{~mm} \mathrm{Hg}$ were remarkably constant. Indeed, analysis of the raw LDF data has shown a clear autoregulatory break point at a cerebral perfusion pressure of $58 \mathrm{~mm} \mathrm{Hg}$, below which cortical perfusion fails. This value is consistent with the autoregulatory breakpoint derived from middle cerebral artery flow velocity waveform analysis obtained with transcranial Doppler studies in other patients with head injuries. ${ }^{45}$ These highly convergent data came as a surprise, and indicate that by contrast with experimental experience with small animals, ${ }^{5172024}$ the methodology adopted seems to provide an LDF baseline reading that is comparable between patients. The use of a skull bolt that excludes interference from external light may be a helping factor. If further experience gives reproducible flux signals in adult brain for a given cerebral blood flow, calibration of the LDF signal may be possible.

\section{SUMMARY}

In summary, early experience with LDF has indicated that long term recordings of cortical perfusion are possible. The reliability of the technique seems to depend on probe positioning and avoidance of movement artifact. An autoregulatory threshold for cortical microcirculatory failure was demonstrated at a cerebral perfusion pressure of $58 \mathrm{~mm} \mathrm{Hg}$, and variations in red cell flux were closely coupled with middle cerebral artery flow velocity measurements in most, but not all, instances. The high temporal resolution of LDF provides the opportunity to monitor the microcirculatory effects of treatment that alters the cerebral perfusion pressure in patients with raised intracranial pressure. Further attention to probe design and the technique of insertion are required for the method to become fully reliable.

1 Marmarou A, Anderson RL, Ward JD, et al. Impact of ICP instability and hypotension in patients with severe head trauma. F Neurosurg 1991;75:859-66.

2 Chan KH, Miller JD, Dearden NM, et al. The effect of changes in cerebral perfusion pressure upon middl cerebral artery blood flow velocity and jugular bulb venous oxygen saturation after severe brain injury. F Neurosurg 1992;77:55-61.

3 Bouma GJ, Muizelaar PJ, Stringer WA, et al. Ultra-early evaluation of regional cerebral blood flow in severely head injured patients using xenon-enhanced computerised tomography. $₹$ Neurosurg 1992;77:360-8.

4 Czosnyka M, Guazzo E, Iyer V, et al. Testing of cerebral autoregulation in head injury by waveform analysis of autoregulation in head injur by brain ed

Kirkatrick PJ Doppler ultrasonography and use of a standardised pulsatility index in the assessmment of head injured patients. I Neurol Neurosurg Psychiatry 1993;57:392.

6 Macpherson P, Graham DI. Arterial spasm and slowing of the cerebral circulation in the ischaemia of head injury. $\mathscr{F}$ Neurol Neurosurg Psychiatry 1973;48:560-4.

7 Aaslid $R$, Lundar $T$, Lindegaard KF, Nornes $H$. Estimation of cerebral perfusion pressure and transcraeds. Intracranial pressure VI Berlin: Springer Verlag, 1986

8 Obrist WD, Wilkinson WE. Regional cerebral blood flow measurement in humans by xenon-133 clearance. Cerebrovasc Brain Metab Rev 1990;2:283-327.

9 Stern MD. In vivo evaluation of microcirculation by coherent light scattering. Nature 1975;254:56-8.

10 Stern MD, Lappe LD, Bowen PD. Continuous Measurement of tissue blood flow by laser-Dopple spectroscopy. Am $\mathcal{F}$ Physiol 1977;232:H441-8.

11 Holloway GA. Laser Doppler measurement of cutaneou blood flow. Non Invasive Measurements 1983;2:220-49.

12 Nilsson GE. Signal processors for laser Doppler tissue flowmeters. Med Biol Eng Comput 1984;22:343-8.

13 Ahn $\mathrm{H}$, Johansson $\mathrm{K}$, Lundgren $\mathrm{O}$, Nilsson GE. In vivo calibration of signal processors for laser Doppler tissue flowmeters. Med Biol Eng Comput 1987;25:207-11.

14 Bonner R, Nossal R. Model for laser Doppler measurements of blood flow in tissue. Applied Optics 1981 20:2097-107.

15 Nilsson GE, Tenland T, Oberg PA. Evaluation of a laser Doppler flowmeter of tissue blood flow. IEEE Trans Biomed Eng 1980;27:579-604.

16 Almond NE, Wheatley AM. Measurement of hepatic perfusion in rats by laser Doppler flowmetry. Am f Physio 1992;262:G203-9.

17 Eyre JA, Essex JTH, Flecknell PA, et al. A comparison of measurements of cerebral blood flow in the rabbit using laser Doppler spectroscopy and radionuclide labelled microspheres. Clin Phys Physiol Meas 1988;9:65-74.

18 Shepherd AP, Riedel GL, Kiel JW, et al. Evaluation of an infrared laser Doppler blood flowmeter. Am F Physio 1987;252:G832-9.

19 Skarphedinsson JO, Harding H, Thoren P. Repeated measurements of cerebral blood flow in rats. Comparisons between the hydrogen clearance method and laser Doppler flowmetry. Acta Physiol Scand 1988;134: 133-42.

20 Dirnagl U, Kaplan B, Jacewicz M, Pulsinelli W. Continuous measurement of cerebral blood flow by laser Continuous measurement of cerebral blood flow by laser Doppler flowmetry in a rat

21 Florence G, Seylaz J. Rapid autoregulation of cerebral blood flow: a laser-Doppler flowmetry study. $f$ Cereb Blood Flow Metab 1992;12:674-80.

22 Goadsby PJ. Characteristics of facial nerve elicited cerebra vasodilation determined using laser Doppler flowmetry. Am F Physiol 1991;260:R255-62.

23 Haberl RL, Heizer ML, Ellis EF. Laser-Doppler assessment of brain microcirculation: effect of systemic alterations. Am F Physiol 1989;256:H1247-54.

24 Haberl RL, Heizer ML, Ellis EF. Laser-Doppler assessment of brain microcirculation: effect of local alterations. Am ₹ Physiol 1989;256:H1255-60.

25 Iadecola C, Reis D. Continuous monitoring of cerebrocortical blood flow during stimulation of the cerebellar fastigial nucleus: A study by laser Doppler flowmetry. fastigial nucleus: A study by laser Doppler

26 Nobes MS, Harris PJ, Yamanda $H$ Mendelsohn FA. Effects of angiotensin on renal cortical and papillary blood flows measured by laser-Doppler flowmetry. $A m \mathcal{F}$ Physiol 1991;261:F998-1006.

27 Skarphedinsson JO, Delle M, Hoffman P, Thoren P. Th effects of naloxone on cerebral blood flow and cerebra function during relative cerebral ichaemia. $f$ Cereb Blood flow Metab 1989;9:515-22.

28 Rendell M, Bergman T, O'Donnell G, et al. Microvascular blood flow, volume, and velocity measured by lase Doppler techniques in IDDM. Diabetes 1989;38: 819-24.

29 Bonard D, Bounameaux H, Fagrell B. Effects of oxygen inhalation on skin microcirculation in patients with peripheral arterial occlusive disease. Circulation peripheral arte

30 Davies EG, Sullivan PM, Fitzpatrick M, Kohner EM Validation and reproducibility of bidirectional laser Validation and reproducibility of bidirectional laser Doppler velocimetry for the measurement
blood flow. Curr Eye Res 1992;11:636-40.

31 Fasano VA, Urciuoli R, Bolognese P, Mostert $M$ Intraoperative use of laser Doppler in the study of cerebral microvascular circulation. Acta Neurochir (Wien) 1988;95:40-8.

32 Rosenblum BR, Bonner RF, Oldfield EH. Intraoperative measurement of cortical blood flow adjacent to cerebra AVM using laser Doppler velocimetry. $f$ Neurosurg 1987;66:396-9.

33 Arbit E, DiResta GR, Bedford, et al. Intraoperative measurement of cerebral and tumour blood flow with lase Doppler flowmetry. Neurosurgery 1989;24:166-70.

34 Meyerson BA, Gunasekera L, Linderoth B, Gazeliu B. Bedside monitoring of regional cortical blood flow in comatose patients using laser Doppler flowmetry. Neurosurg 1991;29:750-5.

35 Richards HK, Czosnyka M, Kirkpatrick PJ, Pickard JD Estimation of laser Doppler flux biological zero using basilar artery flow velocity in the rabbit. Am F Physiol 1994 (in press). 